\title{
Maternal undernutrition leads to endothelial dysfunction in adult male rat offspring independent of postnatal diet
}

\author{
Christopher Torrens ${ }^{1,2 *}$, Mark A. Hanson ${ }^{2}$, Peter D. Gluckman ${ }^{1}$ and Mark H. Vickers ${ }^{1}$ \\ ${ }^{1}$ Liggins Institute, Faculty of Medical and Health Science, University of Auckland and National Research Centre for Growth \\ and Development, Auckland, New Zealand \\ ${ }^{2}$ Centre for Developmental Origins of Health and Disease, Institute of Developmental Sciences, School of Medicine, University of \\ Southampton, Southampton General Hospital, Tremona Road, Southampton SO16 6YD, UK
}

(Received 18 June 2007 - Revised 4 December 2007 - Accepted 20 March 2008 - First published online 20 May 2008)

Increasing evidence suggests a role for prenatal environment in the onset of cardiovascular and metabolic disease in later life. In the rat, undernutrition in utero and a postnatal high-fat diet gives rise to a phenotype similar to the metabolic syndrome. As endothelial dysfunction is a feature of both CVD and the metabolic syndrome we investigated the impact of maternal undernutrition and/or postnatal high-fat on endothelial function. Virgin Wistar rats were mated and randomly assigned to groups to receive food either ad libitum (control) or at $30 \%$ of ad libitum intake throughout gestation. At postnatal day 250, a cohort from each group was challenged with a high-fat diet (D12451, 45\% energy from fat; Research Diets, Inc., New Brunswick, NJ, USA) for the remainder of the study. At 1 year of age, small mesenteric arteries were dissected and mounted on a wire myograph and responses to phenylephrine, endothelin, acetylcholine, leptin and sodium nitroprusside assessed. Vasoconstriction to endothelin was significantly enhanced in all groups compared with controls (-log effective concentration equal to $50 \%$ of the maximal response (pEC 50 ); $P<0.001)$. Endothelium-dependent vasodilatation to acetylcholine was significantly blunted in all groups compared with controls $(\%$ maximum response; $P<0.01$ ), while dilatation to leptin and sodium nitroprusside was similar in all groups. These data demonstrate that both maternal undernutrition and postnatal high fat lead to vascular alterations and suggest that maternal undernutrition alone is at least as detrimental to offspring endothelial function as a long-term exposure to a high-fat diet in the offspring.

Endothelium: Diet: Metabolic syndrome

The classical risk factors for cardiovascular and metabolic disease include smoking, obesity, sedentary lifestyle, high blood pressure and a obesogenic $\operatorname{diet}^{(1)}$. More recently, epidemiological evidence and animal models have demonstrated that the in utero environment may also be an important risk factor for the development of adult disease. Epidemiological and experimental evidence highlights a relationship between the periconceptional, fetal and early infant phases of life and the subsequent development of adult disease. This concept, the 'developmental origins of health and disease' model, proposes that the fetus adapts to adverse environmental cues in utero with permanent readjustments in homeostatic systems to aid survival ${ }^{(2,3)}$. However, these adaptations, known as predictive adaptive responses, may ultimately be disadvantageous in postnatal life and may lead to an increased risk of chronic disease in adulthood ${ }^{(4)}$. More recent evidence has indicated that the greater risk is borne in those who were small at birth but underwent rapid postnatal growth ${ }^{(5)}$.
It has been proposed that the greater the mismatch between the in utero and extra-uterine environment, the greater the risk of adult disease $\mathrm{e}^{(4)}$. These insights have been strengthened by the development of several animal models of unbalanced nutrition in pregnancy which leads to cardiovascular and metabolic dysfunction in the offspring ${ }^{(6)}$. In the rat, restriction of maternal protein or total dietary intake throughout gestation leads to raised blood pressure and endothelial dysfunction in the offspring ${ }^{(7-9)}$. To better describe the interaction between the pre- and postnatal environment we have developed a rat model which gives rise to offspring exhibiting raised blood pressure, obesity, reduced activity, insulin insensitivity, hyperleptinaemia and hyperphagia, particularly in the presence of a postnatal high-fat diet ${ }^{(10-12)}$. The cluster of conditions in this model resembles the human metabolic syndrome, a cluster of metabolic and vascular disorders associated with insulin resistance, hypertension, dyslipidaemia, obesity as well as hyperleptinaemia, microalbuminuria and a range of vascular injury markers ${ }^{(13)}$.

Abbreviations: ACh, acetylcholine; AD, ad libitum; ADC, ad libitum chow-fed; ADHF, ad libitum high-fat-fed; COX, cyclo-oxygenase; EDHF, endothelial-derived hyperpolarising factor; ET, endothelin; KPSS, potassium physiological salt solution; L-NAME, $N_{\omega}$-nitro-L-arginine methyl ester; NOS, NO synthase; $\mathrm{PE}$, phenylephrine; $\mathrm{pEC}_{50}$, - log effective concentration equal to $50 \%$ of the maximal response; $\mathrm{PGI}_{2}$, prostacyclin; UN, undernourished; UNC, undernourished chow-fed; UNHF, undernourished high-fat-fed.

* Corresponding author: Dr Christopher Torrens, fax +44 238079 5255, email c.torrens@southampton.ac.uk 
The vascular endothelium plays an important role in the control of vascular tone, through the release of both vasodilatory factors such as $\mathrm{NO}$, prostacyclin $\left(\mathrm{PGI}_{2}\right)$ and endothelial-derived hyperpolarising factor $(\mathrm{EDHF})^{(14)}$, as well as vasoconstrictors such as endothelin $(\mathrm{ET})^{(15)}$. Endothelial dysfunction is associated with atherosclerosis $^{(16)}$, hypertension ${ }^{(17)}$ but is also a common feature of the metabolic syndrome ${ }^{(18)}$. The aim of the present study was to determine whether vascular dysfunction was also present in offspring following developmental programming, and, if so, whether it was exacerbated by a postnatal high-fat dietary challenge on vascular function.

\section{Methods}

A previously developed maternal undernutrition model of developmental programming was utilised in the present study ${ }^{(10)}$. In brief, virgin Wistar rats (age $100 \pm 5 \mathrm{~d}$ ) were time-mated using a rat oestrous cycle monitor to assess the stage of oestrous of the animals before introducing the male. After confirmation of mating, rats were housed individually in standard rat cages with free access to water. Rats were kept in the same room with a constant temperature maintained at $25^{\circ} \mathrm{C}$ and a $12 \mathrm{~h}$ light $-12 \mathrm{~h}$ darkness cycle. Animals were assigned to either a control diet of ad libitum intake throughout gestation (AD; $n 16)$ or an undernutrition diet (UN, $30 \%$ of ad libitum; $n$ 16). Food intake and maternal weights were recorded daily until birth. After birth, pups were weighed, and litter size was recorded. Pups from undernourished mothers were cross-fostered onto dams that received ad libitum feeding throughout pregnancy. Litter size was adjusted to eight pups per litter to ensure adequate and standardised nutrition until weaning. At weaning, male AD and UN offspring were placed on a standard rat chow (Harlan Teklad Diet 2018; Harlan Teklad, Bicester, Oxon, UK) fed ad libitum (ADC, UNC). At postnatal day 240, a cohort from each group was challenged with a high-fat diet (D12451, $45 \%$ energy from fat; Research Diets, Inc., New Brunswick, NJ, USA) for the remainder of the study (ADHF, UNHF). The fat source in the high-fat diet was derived from lard $(39.5 \%$ of total energy) and soyabean oil (5.5\% of total energy) with fat content by weight 5 and $24 \%$ for the chow and high-fat diets respectively. The energy density of the chow diet was $14.2 \mathrm{~kJ} / \mathrm{g}$ compared with $19.8 \mathrm{~kJ} / \mathrm{g}$ for the high-fat diet. The mineral and vitamin content in the two diets was identical and in accordance with the requirements for standard rat diets. At age $365 \mathrm{~d}$, rats were euthanised with pentobarbitone $(60 \mathrm{mg} / \mathrm{kg})$ followed by decapitation. All animal procedures were approved by The Animal Ethics Committee of the University of Auckland.

\section{Vascular protocol - adult offspring mesenteric arteries}

Mesenteric artery segments (internal diameter about $300 \mu \mathrm{m}$ ) were dissected, cleaned of connective tissue and mounted on a wire myograph (Danish Myo Technology A/S, Aarhus, Denmark). Segments were bathed in physiological salt solution of the following composition: $\mathrm{NaCl}, 119 ; \mathrm{KCl}, 4 \cdot 7 ; \mathrm{CaCl}_{2}$, 2.5; $\mathrm{MgSO}_{4}, 1 \cdot 17 ; \mathrm{NaHCO}_{3}, 25 ; \mathrm{KH}_{2} \mathrm{PO}_{4}, 1 \cdot 18$; EDTA, 0.026; D-glucose, $5.5 \mathrm{~mm}$, heated to $37^{\circ} \mathrm{C}$ and continuously gassed with $95 \% \mathrm{O}_{2}$ and $5 \% \mathrm{CO}_{2}$. The passive tension-internal circumference relationship was determined by incremental increases in tension to achieve an internal circumference equivalent to a transmural pressure of $100 \mathrm{mmHg}\left(\mathrm{IC}_{100}\right)$ using the
Laplace relationship. Arteries were set to a diameter equivalent to $0.9 \times \mathrm{IC}_{100}$ as previously described ${ }^{(19)}$. Functional integrity of the smooth muscle was assessed with four 2 min washes with $125 \mathrm{~mm}$-KPSS solution (physiological salt solution with an equimolar substitution of $\mathrm{KCl}$ for $\mathrm{NaCl}$ ). Vessels failing to produce an active tension equivalent to $13.3 \mathrm{kPa}$ were discarded from the study.

Following normalisation and tests of functional integrity, cumulative concentration-response curves were constructed for the $\alpha_{1}$-adrenoceptor agonist phenylephrine (PE; $10 \mathrm{nM}$ to $100 \mu \mathrm{M})$ and ET (1 pM to $10 \mathrm{nM})$. Then, following pre-constriction with $\mathrm{PE}$ (-log effective concentration equal to $80 \%$ of the maximal response; $\mathrm{pEC}_{80}$ ), cumulative concentrationresponse curves were constructed to the endothelium-dependent vasodilator acetylcholine (ACh; $0 \cdot 1 \mathrm{nM}$ to $10 \mu \mathrm{M}$ ), the hormone leptin $(0.01$ to $10 \mathrm{ng} / \mathrm{ml})$ and the NO donor sodium nitroprusside $(0 \cdot 1 \mathrm{nM}$ to $10 \mu \mathrm{M})$. To investigate the factors involved in ACh-mediated vasodilatation, responses to ACh were repeated in vessels incubated with the non-selective NO synthase (NOS) inhibitor $N_{\omega}$-nitro-L-arginine methyl ester (L-NAME; $100 \mu \mathrm{M}$ ) and the cyclo-oxygenase (COX) inhibitor indomethacin $(10 \mu \mathrm{M}) 30 \mathrm{~min}$ before commencing the ACh response. These inhibitors were given only in combination and not independently, based on our previous experience that the $\mathrm{PGI}_{2}$ and therefore COX-sensitive component of the ACh response is negligible in this particular vascular bed (C Torrens, unpublished results). Leptin was investigated due to our previous observation that early acute leptin treatment could reverse the effects of the diets ${ }^{(12)}$. Leptin has previously been shown to be an endothelial-dependent vasodilator in isolated arteries ${ }^{(20)}$, and we also investigated responses to leptin in arterial segments before and following pre-incubation with L-NAME $(100 \mu \mathrm{M})$ for $30 \mathrm{~min}$.

All drugs and chemicals were obtained from Sigma-Aldrich (Auckland, New Zealand) with the exception of recombinant rat leptin (Arieh Gertler, University of Rehovot, Israel).

\section{Calculations and statistical analysis}

Data are expressed as mean values with their standard errors. Constrictor responses were calculated as percentage of maximum contraction induced by $125 \mathrm{mM}$-KPSS and relaxant responses as percentage inhibition of PE-induced contraction. Cumulative concentration-response curves to agonists were analysed by fitting to a four-parameter logistic equation using non-linear regression to obtain the -log effective concentration equal to $50 \%$ of the maximal response $\left(\mathrm{pEC}_{50}\right)$ and maximum response. Differences were assessed by one-way ANOVA with Bonferroni post hoc correction. When the curve produced by non-linear regression was dissimilar to the unfitted data, curve-fitted data were not used. Where curves were not sigmoidal, calculation of the $\mathrm{pEC}_{50}$ was deemed inappropriate and concentration-response curves were compared using two-way ANOVA. Significance was accepted at the $P<0.05$ level.

\section{Results}

\section{Maternal undernutrition}

Maternal undernutrition resulted in fetal growth retardation reflected by significantly decreased birth weight (AD, 6.05 
(SEM 0.01) g; UN, 4.34 (SEM 0.02) g; $P<0 \cdot 0001$ ) and nose-anus length (AD, 48.6 (SEM 0.01) mm; UN, $41 \cdot 3$ (SEM 0.01) mm; $P<0.0001)$ in the offspring from UN dams. Litter size was not different between the two groups (AD, 12.3 (SEM 1.8); UN, 11.9 (SEM 2.0)). UN animals remained significantly lighter and shorter than $\mathrm{AD}$ animals for the duration of the trial.

\section{Effect of postnatal high-fat dietary challenge}

At postnatal day 240, animals were weight matched within group and placed on either chow or a high-fat diet $(45 \%$ energy as fat, Research Diets D12451; Research Diets, Inc., New Brunswick, NJ, USA) fed ad libitum for the remainder of the trial (day 365). Total body-weight gain was significantly increased in all high-fat-fed animals (ADC, 799 (SEM 10) g; ADHF, 977 (SEM 39) g; UNC, 706 (SEM 24) g; UNHF, 864 (SEM 26) g) and there were no differences in the susceptibility to diet-induced weight gain in UN animals on the high-fat diet compared with $\mathrm{AD}$ animals (Fig. 1). There was a small but significant increase $(P<0.05)$ in energy intake in UN animals compared with $\mathrm{AD}$ animals and a significant increase $(P<0 \cdot 001)$ in total energy intake in high-fat-fed animals compared with chow-fed offspring ( $\mathrm{kJ} / \mathrm{g}$ body weight; ADC, 0.540 (SEM 0.008); ADHF, 0.623 (SEM 0.013); UNC, 0.586 (SEM 0.013); UNHF, 0.653 (SEM 0.008)).

\section{Mesenteric artery reactivity}

In all arteries the depolarising KPSS wash produced a vasoconstriction that did not differ between the four groups (data not shown). The $\alpha_{1}$-adrenoceptor agonist PE produced a concentration-dependent vasoconstriction in all arteries. In both groups of animals fed the high-fat diet, sensitivity to PE was significantly reduced compared with controls; this was independent of the earlier challenge and was not different to the UN chow response ( $\mathrm{pEC}_{50}$; ADC, 5.90 (SEM 0.04) ( $n$ 7); ADHF, 5.67 (SEM 0.04) ( $n$ 8); UNC, 5.81 (SEM 0.04) ( $n$ 8); UNHF, 5.70 (SEM 0.05) ( $n$ 8); $P<0.05$; Fig. 2 (A)).

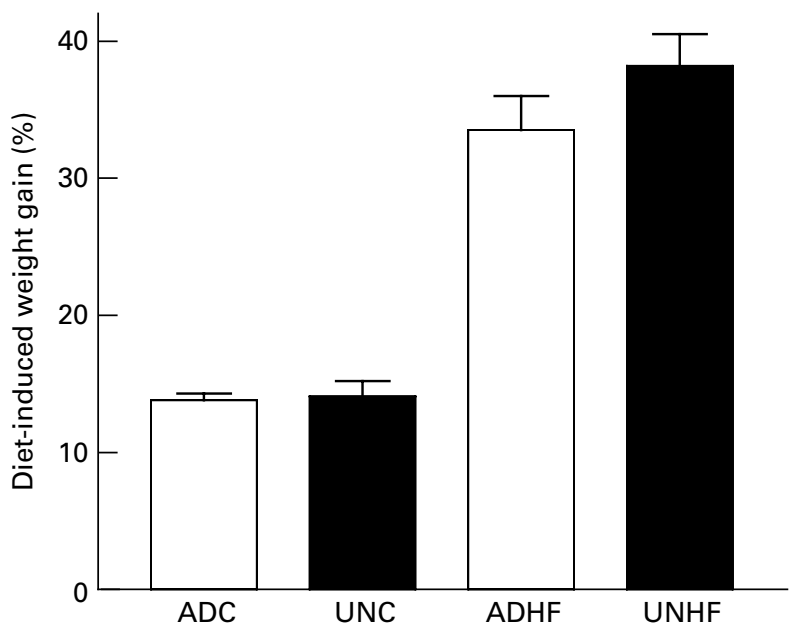

Fig. 1. Diet-induced body-weight gain (\%) in ad libitum-fed (AD) and undernourished (UN) animals fed either chow (C) or challenged with a high-fat (HF) diet from day 240 until the end of the trial. Data are means for twelve animals per group, with standard errors represented by vertical bars. The effect of the HF diet was significant $(P<0.0001)$.
Similarly, $\mathrm{ET}_{1}$ produced a potent vasoconstriction in all arteries, but which was significantly more potent in each of the three treatment groups compared with the controls $\left(\mathrm{pEC}_{50} ; \mathrm{ADC}, 9.30\right.$ (SEM 0.07) ( $n$ 7); ADHF, 9.79 (SEM 0.11) $(n$ ) $)$ UNC, 10.01 (SEM 0.11) ( $n$ 8); UNHF, 10.02 (SEM 0.15) (n 8); $P<0.01$; Fig. 2 (B)).

\section{Endothelial-dependent vasodilatation}

In all groups the endothelial-dependent vasodilator $\mathrm{ACh}$ produced a concentration-dependent vasodilatation. In all groups vasodilatation to $\mathrm{ACh}$ was significantly impaired compared with the AD group (\% maximum response; ADC, 72.4 (SEM 3.3) ( $n$ 7); ADHF, 47.5 (SEM 4.4) ( $n$ 8); UNC, 44.4 (SEM 4.4) ( $n$ 8); UNHF, 45.5 (SEM 3.3) $(n$ 8); $P<0.01$; Fig. 3 (A)).

ACh responses in the presence of the NOS inhibitor L-NAME $(100 \mu \mathrm{M})$ and the COX inhibitor indomethacin $(10 \mu \mathrm{M})$ were significantly impaired compared with naive preparations in all groups $(P<0.001$; Fig. $3(\mathrm{~B}))$. In the presence of the inhibitors, the previously observed differences in the $\mathrm{ACh}$ response between the groups were abolished. Moreover, the size of response to ACh following NOS and COX blockade would indicate a sizeable $\mathrm{NO} / \mathrm{PGI}_{2}$ component with a relatively poor EDHF component.

\section{Leptin-induced vasodilatation}

In all groups, leptin produced a weak inhibition of PE-induced tone that did not differ between the groups (Fig. 4 (A)). What dilatation there was observed to leptin was unaffected in a subgroup of arteries ( $n 3)$, when repeated in the presence of the NOS inhibitor L-NAME $(100 \mu \mathrm{M})$ in all groups (data not shown).

\section{Endothelium-independent vasodilatation}

In all groups, the NO donor sodium nitroprusside produced a concentration-dependent vasodilatation in all four experimental groups (Fig. 4 (B)).

\section{Discussion}

An adverse intra-uterine environment is associated with longterm metabolic consequences, in particular obesity and CVD. Data from epidemiological as well as animal studies have given rise to the concept of developmental programming, whereby an unfavourable prenatal environment is believed to trigger adaptive responses that improve fetal survival or prepare the fetus in expectation of a particular range of environments postnatally. However, if the pre- and postnatal environments are widely discrepant, these adaptive responses may prove to be the origins of later disease ${ }^{(4)}$. Our model of maternal undernutrition followed by postnatal high fat ${ }^{(10-12)}$ supports epidemiological evidence that the greatest risk is seen in those who are born small but have rapid postnatal weight gain $^{(5)}$. The present study shows that endothelial dysfunction is manifest in offspring of undernourished mothers, independently of postnatal dietary environment and is a permanent consequence of adverse prenatal conditions. 

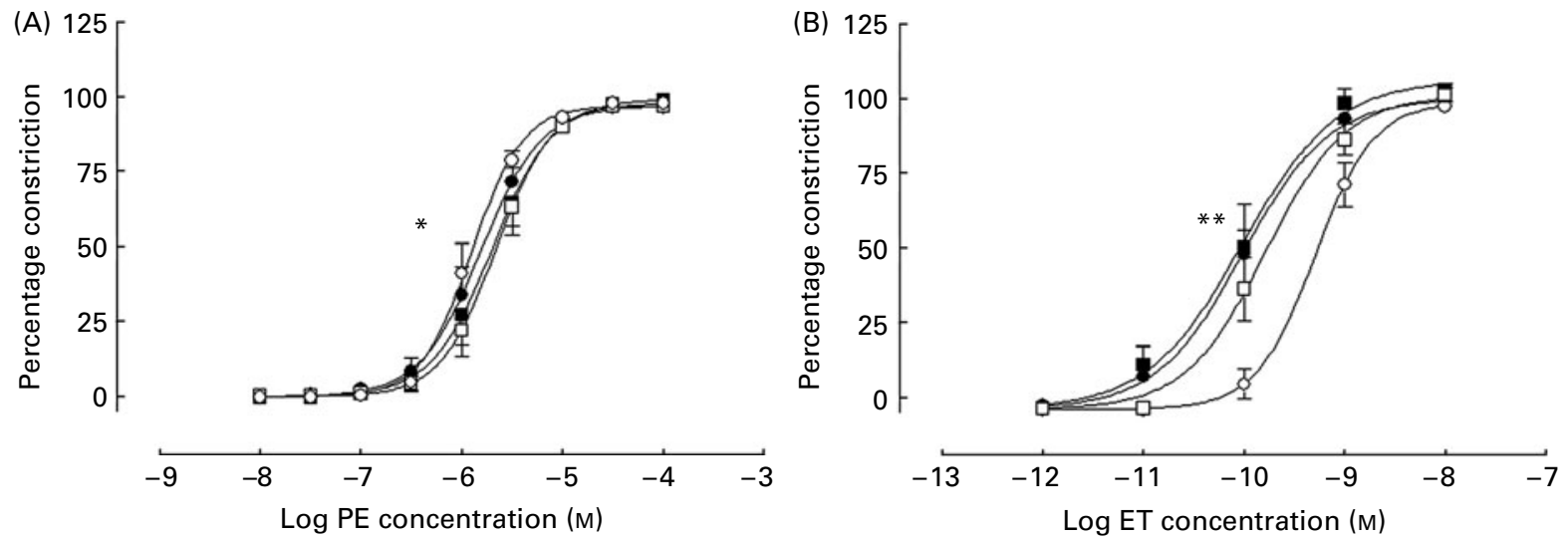

Fig. 2. Cumulative additions of $(A)$ the $\alpha_{1}$-adrenoceptor agonist phenylephrine (PE) and $(B)$ endothelin (ET) to mesenteric arteries of 365 - $d$-old male rats from

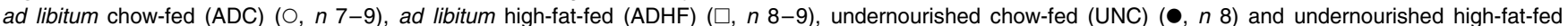
(UNHF) (ם,n 7-9) groups. Data are means, with standard errors represented by vertical bars. * Mean value of -log effective concentration equal to $50 \%$ of the maximal response $\left(\mathrm{pEC}_{50}\right)$ for the $\mathrm{AD}$ rats was significantly different from that for the ADHF and UNHF rats $(P<0.05)$. ${ }^{* \star}$ Mean value of $\mathrm{pEC} \mathrm{F}_{50}$ for the $\mathrm{ADC}$ rats was significantly different from that for the ADHF, UNC and UNHF rats $(P<0.01)$.

The vascular endothelium is important in the control of vascular tone, and endothelial dysfunction is a common feature of atherosclerosis ${ }^{(16)}$ and hypertension ${ }^{(17)}$ as well as type 2 diabetes and the metabolic syndrome ${ }^{(18)}$. Impaired endothelial function following maternal dietary manipulations have been previously reported in the offspring of fat-fed dams ${ }^{(21-23)}$ as well as offspring from protein-restricted dams ${ }^{(7,9)}$. Similarly to the present study, endothelial dysfunction has also been seen in the offspring of dams following global nutrient restriction. However, these have not always been consistent and may depend on the severity and duration of the challenge as to whether effects on endothelial function are seen ${ }^{(8,24)}$, or not ${ }^{(25)}$. While evidence for altered endothelial function exists from the offspring of nutrient-restricted dams, the present study is the first to examine the possible effects of nutritional mismatches on postnatal endothelial function.

A diet high in fat has long been considered a risk factor for such CVD, and the impact of high-fat diets on blood pressure and vascular function is well documented ${ }^{(26-29)}$. The present study confirms these observations, noting that endothelial function is impaired following a high-fat diet. In addition, the relative hyperphagia and predisposition to sedentary activity in this model may have contributed to the endothelial dysfunction observed in offspring of undernourished mothers ${ }^{(10,11)}$. We have previously described increased systolic blood pressure in a similar animal model ${ }^{(10-12)}$ and, taken together, our finding of endothelial dysfunction may not be surprising. What is interesting about these findings, however, is whilst the effects on systolic blood pressure showed a synergistic interaction between the maternal undernutrition and postnatal diet ${ }^{(10-12)}$, no such interaction was observed in reference to endothelial function. The lack of such an interaction in the present study may suggest that the timing of the postnatal challenge is important, as the postnatal high-fat feeding began later in the present study compared with previous studies. However, despite the shorter duration of the high-fat challenge in the present study it is clear that the energy intake and weight gain was still significantly greater in the high-fat groups, suggesting that the pre-pubertal timing in previous reports may be of importance to the synergistic effects of
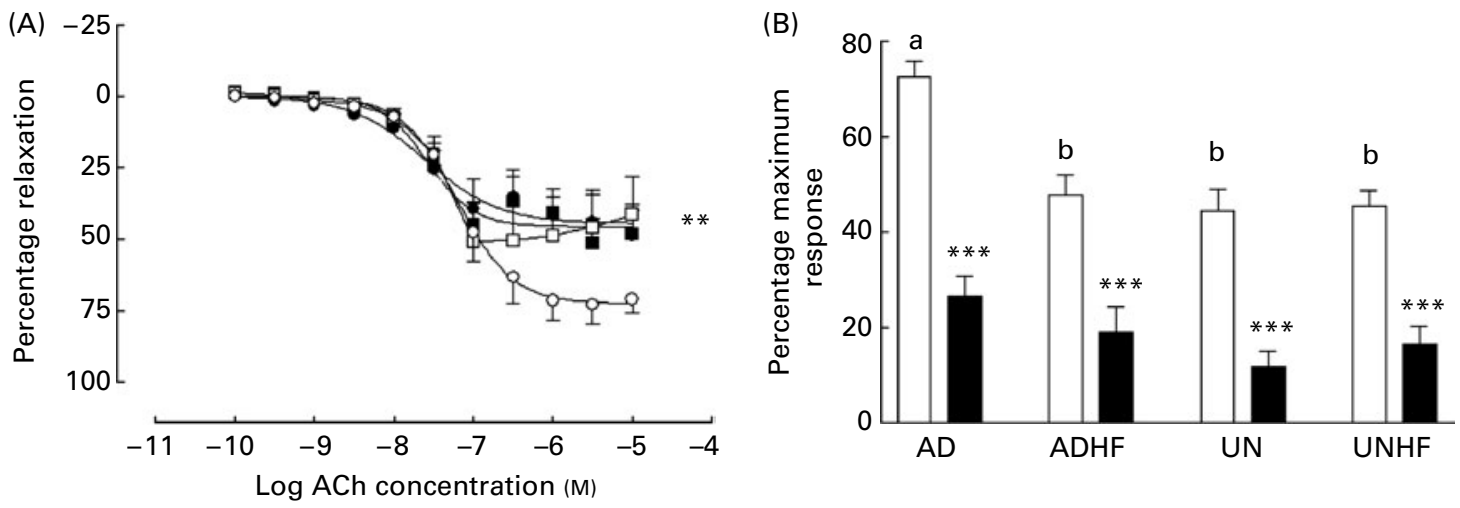

Fig. 3. (A) Cumulative additions of the endothelial-dependent vasodilator acetylcholine (ACh) to mesenteric arteries of 365 -d-old rats from ad libitum chow-fed $(\mathrm{ADC})(\mathrm{O}, n \mathrm{7})$, ad libitum high-fat-fed (ADHF) ( $\square, n$ 8), undernourished chow-fed (UNC) $(\bullet, n$ 8) and undernourished high-fat-fed (UNHF) ( $\mathbf{\square}, n$ 8) groups. Data are means, with standard errors represented by vertical bars. ${ }^{* *}$ Mean value of percentage maximum response for the ADC rats was significantly different from that for the ADHF, UNC and UNHF rats $(P<0.01)$. (B) Maximal response to ACh in small mesenteric arteries in the absence $(\square, n 7-9)$ and presence of $N_{\omega}-$ nitroL-arginine methyl ester $(100 \mu \mathrm{M})$ and indomethacin $(10 \mu \mathrm{M})(\boldsymbol{\square}, n 6-8)$. Data are means, with standard errors represented by vertical bars. ${ }^{\star \star \star}$ Mean value was significantly different from that of the naive preparation $(P<0.001) .{ }^{\text {ab }}$ Mean values with unlike letters were significantly different $(P<0.01)$. 

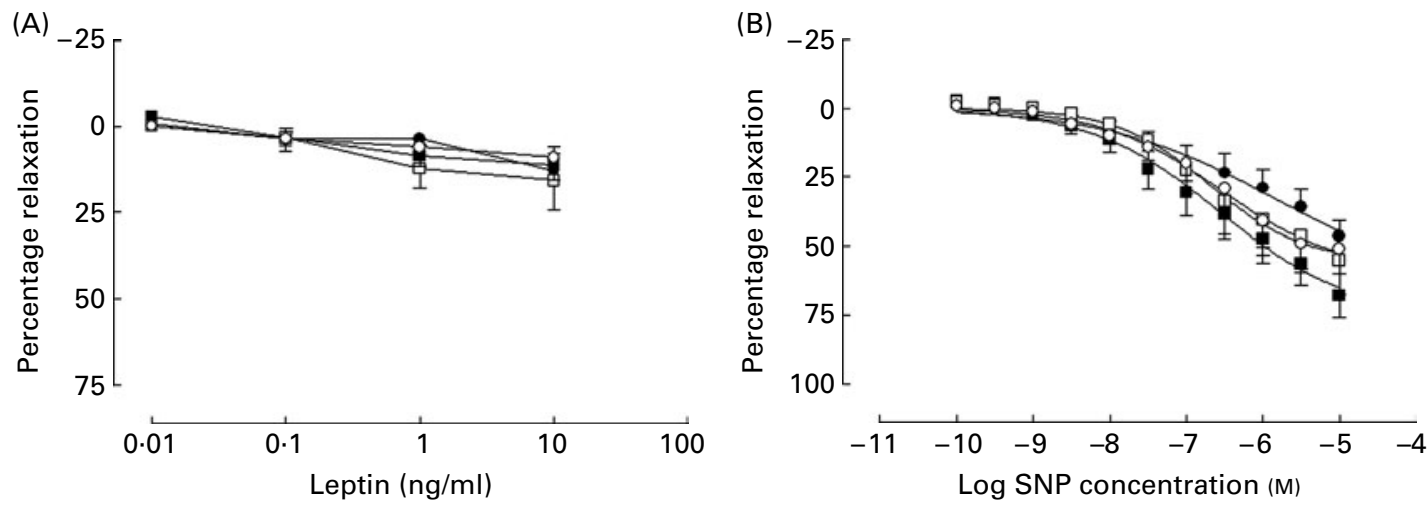

Fig. 4. Cumulative additions of $(A)$ the appetite hormone leptin and (B) the NO donor sodium nitroprusside (SNP) to mesenteric arteries from $365-\mathrm{d}$-old rats from ad libitum chow-fed $(O, n 5-6)$, ad libitum high-fat-fed $(\square, n 7)$, undernourished chow-fed $(\bullet, n 5-8)$ and undernourished high-fat-fed $(\boldsymbol{\square}, n$ 6-8) rats. Data are means, with standard errors represented by vertical bars.

pre- and postnatal dietary interactions ${ }^{(12)}$. Alternatively, it is possible that postnatal high-fat diets and maternal undernutrition have comparable effects on the vascular endothelium and the mediators of vasodilatation and that whilst postnatal diet and lifestyle have an undoubted effect on cardiovascular health, the prenatal environment may be of equal importance.

Our evidence of endothelial dysfunction is inferred from our observation of an attenuated dilatation to $\mathrm{ACh}$ in all three experimental groups at 1 year of age. In rat small mesenteric arteries, ACh-mediated vasodilatation involves the release of $\mathrm{NO}, \mathrm{PGI}_{2}$ and EDHF from the endothelium ${ }^{(14)}$, with EDHF providing the major contribution, a smaller, yet sizeable NO component and only a small $\mathrm{PGI}_{2}$ component ${ }^{(23)}$. As such, the impaired dilatation reported in the present study is likely to be due to alteration in one or more of these pathways, and experiments were conducted in the presence of NOS and COX blockade to identify the component parts of the ACh response. There are two interesting findings from the more in-depth analysis of the ACh response. First, it is clear that following NOS and COX blockade, the response to ACh was almost abolished, indicating a relatively small EDHF component in comparison with younger animals. Second, the differences previously noted in the ACh response are no longer present. Taken together, these data indicate that the EDHF component is equivalent in all groups and, given the minor role for $\mathrm{PGI}_{2}$ in these arteries, the impairment in the ACh response is likely to be due to decrease in the NO pathway.

A decrease in the bioavailability NO could arise from a decrease in the endothelial NOS expression or activity as well as an increase in oxidative stress. Decreased levels of the enzyme endothelial NOS have been reported following maternal undernutrition ${ }^{(9,30)}$ and postnatal high-fat diets ${ }^{(28,29)}$, while increased oxidative stress has been proposed as a mechanism for the vascular dysfunction following maternal undernutrition $^{(8)}$. Previous studies suggest that decreased NO bioavailability enhances the response to sodium nitroprusside, both in the thoracic aorta of endothelial NOS-null mice ${ }^{(31)}$, and also alongside endothelial dysfunction in the offspring of nutrient-restricted dams ${ }^{(24)}$. The present study did not note any change in response to sodium nitroprusside indicating that whilst endothelial-dependent vasodilatation is attenuated, the smooth muscle soluble guanylate cyclase pathway is unaltered by the dietary challenges. In addition, the adipokine leptin has also been suggested to induce vasodilatation through an endothelial-dependent mechanism in isolated arteries ${ }^{(20)}$. In the present study, leptin-induced vasodilatation was poor in comparison with the other vasodilators tested, although previous reports do not indicate leptin to be a particularly potent dilator $^{(20,32)}$. Whilst some have reported leptin acting through an NO-dependent pathway ${ }^{(32)}$, in the mesenteric arteries akin to those investigated in the present study this appears to be an NO-independent, EDHF pathway ${ }^{(20)}$. Owing to the poor response to leptin, only a small number of leptin responses were repeated in the presence of L-NAME ( $n 3$ from all groups). Where performed, NOS blockade had no effect on the leptin response and would seem to suggest that leptin response is NO-independent; the lack of difference between the groups would therefore not contradict a decrease in NO bioavailability.

A novel finding from the present study is the enhanced vasoconstriction to $\mathrm{ET}_{1} . \mathrm{ET}_{1}$ is a potent vasoconstrictor peptide released from the endothelium ${ }^{(15)}$ which acts on two receptor subtypes: $\mathrm{ET}_{\mathrm{A}}$ and $\mathrm{ET}_{\mathrm{B}}$. Whilst the $\mathrm{ET}_{\mathrm{A}}$ mediates constriction, the $\mathrm{ET}_{\mathrm{B}}$ receptor mediates vasodilatation through $\mathrm{NO}^{(33)}$ and can modulate the $\mathrm{ET}_{\mathrm{A}}$-mediated constrictor response ${ }^{(34)}$. As such, the enhanced constriction could be explained by loss of this balance. Alternatively, the endothelium also releases a number of constricting factors including $\mathrm{ET}_{1}$ and thromboxane in addition to the endothelial-derived relaxing factors ${ }^{(14)}$, the enhanced release of which may also be responsible. Both hyperinsulinaemia ${ }^{(35)}$ and hypercholesterolaemia ${ }^{(29,36)}$ have been shown to increase release of $\mathrm{ET}_{1}$. Such enhanced sensitivity to vasoconstrictors following a high-fat diet would also fit with our observation of enhanced PE constriction in both the ADHF and UNHF groups, although not the UNC group. However, while enhanced $\mathrm{ET}_{1}$ release has been implicated in altered $\mathrm{ACh}$ responses observed in aortas of dyslipidaemic rabbits, in contrast to the present study responses to exogenous $\mathrm{ET}_{1}$ were similar ${ }^{(37)}$.

In summary, our data demonstrate that undernutrition in utero and/or a high-fat diet post-weaning leads to endothelial dysfunction in the offspring at 1 year of age. Interestingly, the present data suggest that while each challenge in isolation is detrimental to endothelial function, the effects are 
not cumulative when the offspring are subjected to both challenges. Importantly, while these data support the traditional high-fat risk factor for endothelial dysfunction they also suggest that poor maternal nutrition could be as detrimental to endothelial function and an equivalent risk factor.

\section{Acknowledgements}

C. T. and M. H. V. performed the experimental work. C. T., M. A. H., P. D. G. and M. H. V. wrote the paper. P. D. G. and M. H. V. designed the experiment. Financial support for the study was provided by the National Research Centre for Growth and Development (M. H. V. and P. D. G.). C. T. and M. A. H. received salary support from the British Heart Foundation. There are no conflicts of interest.

\section{References}

1. Forouhi NG \& Sattar N (2006) CVD risk factors and ethnicity a homogeneous relationship? Atheroscler Suppl 7, 11-19.

2. Osmond C, Barker DJ, Winter PD, Fall CH \& Simmonds SJ (1993) Early growth and death from cardiovascular disease in women. BMJ 307, 1519-1524.

3. Kajantie E, Osmond C, Barker DJ, Forsen T, Phillips DI \& Eriksson JG (2005) Size at birth as a predictor of mortality in adulthood: a follow-up of 350000 person-years. Int J Epidemiol 34, 655-663.

4. Gluckman PD \& Hanson MA (2004) Living with the past: evolution, development, and patterns of disease. Science 305, $1733-1736$.

5. Eriksson JG, Forsen T, Tuomilehto J, Osmond C \& Barker DJ (2003) Early adiposity rebound in childhood and risk of type 2 diabetes in adult life. Diabetologia 46, 190-194.

6. McMillen IC \& Robinson JS (2005) Developmental origins of the metabolic syndrome: prediction, plasticity, and programming. Physiol Rev 85, 571-633.

7. Brawley L, Itoh S, Torrens C, Barker A, Bertram C, Poston L \& Hanson M (2003) Dietary protein restriction in pregnancy induces hypertension and vascular defects in rat male offspring. Pediatr Res 54, 83-90.

8. Franco MC, Fortes ZB, Akamine EH, et al. (2004) Tetrahydrobiopterin improves endothelial dysfunction and vascular oxidative stress in microvessels of intrauterine undernourished rats. J Physiol 558, 239-248.

9. Torrens C, Brawley L, Anthony FW, Dance CS, Dunn R, Jackson AA, Poston L \& Hanson MA (2006) Folate supplementation during pregnancy improves offspring cardiovascular dysfunction induced by protein restriction. Hypertension 47, 982-987.

10. Vickers MH, Breier BH, Cutfield WS, Hofman PL \& Gluckman PD (2000) Fetal origins of hyperphagia, obesity, and hypertension and postnatal amplification by hypercaloric nutrition. Am J Physiol Endocrinol Metab 279, E83-E87.

11. Vickers MH, Breier BH, McCarthy D \& Gluckman PD (2003) Sedentary behavior during postnatal life is determined by the prenatal environment and exacerbated by postnatal hypercaloric nutrition. Am J Physiol Regul Integr Comp Physiol 285, R271-R273.

12. Vickers MH, Gluckman PD, Coveny AH, Hofman PL, Cutfield WS, Gertler A, Breier BH \& Harris M (2005) Neonatal leptin treatment reverses developmental programming. Endocrinology 146, 4211-4216.

13. Bonora $\mathrm{E}$ (2006) The metabolic syndrome and cardiovascular disease. Ann Med 38, 64-80.
14. Busse R \& Fleming I (2006) Vascular endothelium and blood flow. Handb Exp Pharmacol 43-78.

15. Yanagisawa $M$, Kurihara $H$, Kimura $S$, Tomobe $Y$, Kobayashi M, Mitsui Y, Yazaki Y, Goto K \& Masaki T (1988) A novel potent vasoconstrictor peptide produced by vascular endothelial cells. Nature 332, 411-415.

16. Landmesser U, Hornig B \& Drexler H (2004) Endothelial function: a critical determinant in atherosclerosis? Circulation 109, II27-II33.

17. Panza JA, Casino PR, Kilcoyne CM \& Quyyumi AA (1993) Role of endothelium-derived nitric oxide in the abnormal endothelium-dependent vascular relaxation of patients with essential hypertension. Circulation 87, 1468-1474.

18. Fornoni A \& Raij L (2005) Metabolic syndrome and endothelial dysfunction. Curr Hypertens Rep 7, 88-95.

19. Mulvany MJ \& Halpern W (1977) Contractile properties of small arterial resistance vessels in spontaneously hypertensive and normotensive rats. Circ Res 41, 19-26.

20. Lembo G, Vecchione C, Fratta L, Marino G, Trimarco V, d'Amati G \& Trimarco B (2000) Leptin induces direct vasodilation through distinct endothelial mechanisms. Diabetes 49, 293-297.

21. Khan IY, Taylor PD, Dekou V, Seed PT, Lakasing L, Graham D, Dominiczak AF, Hanson MA \& Poston L (2003) Gender-linked hypertension in offspring of lard-fed pregnant rats. Hypertension 41, 168-175.

22. Khan I, Dekou V, Hanson M, Poston L \& Taylor P (2004) Predictive adaptive responses to maternal high-fat diet prevent endothelial dysfunction but not hypertension in adult rat offspring. Circulation 110, 1097-1102.

23. Taylor PD, Khan IY, Hanson MA \& Poston L (2004) Impaired EDHF-mediated vasodilatation in adult offspring of rats exposed to a fat-rich diet in pregnancy. J Physiol 558, 943-951.

24. Holemans K, Gerber R, Meurrens K, De Clerck F, Poston L \& Van Assche FA (1999) Maternal food restriction in the second half of pregnancy affects vascular function but not blood pressure of rat female offspring. Br J Nutr 81, 73-79.

25. Williams SJ, Hemmings DG, Mitchell JM, McMillen IC \& Davidge ST (2005) Effects of maternal hypoxia or nutrient restriction during pregnancy on endothelial function in adult male rat offspring. J Physiol 565, 125-135.

26. Gerber RT, Holemans K, O'Brien-Coker I, Mallet AI, van Bree R, Van Assche FA \& Poston L (1999) Cholesterol-independent endothelial dysfunction in virgin and pregnant rats fed a diet high in saturated fat. J Physiol 517, 607-616.

27. Rizzoni D, Porteri E, Guelfi D, et al. (2001) Endothelial dysfunction in small resistance arteries of patients with non-insulin-dependent diabetes mellitus. J Hypertens 19, 913-919.

28. Roberts CK, Barnard RJ, Sindhu RK, Jurczak M, Ehdaie A \& Vaziri ND (2005) A high-fat, refined-carbohydrate diet induces endothelial dysfunction and oxidant/antioxidant imbalance and depresses NOS protein expression. J Appl Physiol 98, 203-210.

29. Song GY, Gao Y, Di YW, Pan LL, Zhou Y \& Ye JM (2006) High-fat feeding reduces endothelium-dependent vasodilation in rats: differential mechanisms for saturated and unsaturated fatty acids? Clin Exp Pharmacol Physiol 33, 708-713.

30. Franco MC, Arruda RM, Dantas AP, Kawamoto EM, Fortes ZB, Scavone C, Carvalho MH, Tostes RC \& Nigro D (2002) Intrauterine undernutrition: expression and activity of the endothelial nitric oxide synthase in male and female adult offspring. Cardiovasc Res 56, 145-153.

31. Waldron GJ, Ding H, Lovren F, Kubes P \& Triggle CR (1999) Acetylcholine-induced relaxation of peripheral arteries isolated from mice lacking endothelial nitric oxide synthase. BrJPharmacol 128, 653-658.

32. Jaffar MM, Myers DS, Hainsworth LJ, Hainsworth R \& Drinkhill MJ (2005) Effects of dietary salt loading on the 
responses of isolated rat mesenteric arteries to leptin. $A m$ J Hypertens 18, 500-503.

33. Fujitani Y, Ueda H, Okada T, Urade Y \& Karaki H (1993) A selective agonist of endothelin type B receptor, IRL 1620, stimulates cyclic GMP increase via nitric oxide formation in rat aorta. J Pharmacol Exp Ther 267, 683-689.

34. Goddard J, Johnston NR, Hand MF, Cumming AD, Rabelink TJ, Rankin AJ \& Webb DJ (2004) Endothelin-A receptor antagonism reduces blood pressure and increases renal blood flow in hypertensive patients with chronic renal failure: a comparison of selective and combined endothelin receptor blockade. Circulation 109, $1186-1193$
35. Piatti PM, Monti LD, Conti M, Baruffaldi L, Galli L, Phan CV, Guazzini B, Pontiroli AE \& Pozza G (1996) Hypertriglyceridemia and hyperinsulinemia are potent inducers of endothelin-1 release in humans. Diabetes 45, 316-321.

36. Lerman A, Edwards BS, Hallett JW, Heublein DM, Sandberg SM \& Burnett JC Jr (1991) Circulating and tissue endothelin immunoreactivity in advanced atherosclerosis. $N$ Engl $\mathrm{J} \mathrm{Med}$ 325, $997-1001$.

37. Maeso R, Aragoncillo P, Navarro-Cid J, Ruilope LM, Diaz C, Hernandez G, Lahera V \& Cachofeiro V (2000) Effect of atorvastatin on endothelium-dependent constrictor factors in dyslipidemic rabbits. Gen Pharmacol 34, 263-272. 